\title{
Parentalidad y salud mental adolescente: diferencias entre ciudades y tipo de dependencia escolar ${ }^{1}$
}

\author{
Parenthood and Adolescence mental health. \\ Differences by Geographic Location and school belonging
}

\author{
Martin Casassus Rodino ${ }^{2}$ \\ Macarena Valdés Correa ${ }^{3}$ \\ Ramón Florenzano Urzúa ${ }^{4}$ \\ Eugenio Cáceres Contreras 5 \\ Carolina Aspillaga Herrera ${ }^{6}$ \\ Sylvia Santander Rigollet ${ }^{7}$
}

Resumen

Los estilos de crianza influyen en la salud mental adolescente. Trabajos previos han corroborado el modelo explicativo de Brian Barber que relaciona variables parentales (control psicológico, control conductual, apoyo social) con otras de funcionamiento adolescente (depresión, comportamiento antisocial, iniciativa social). Este estudio pretende comprobar el modelo en tres ciudades de Chile y tipos de escuelas. Resultados: el modelo responde en la muestra total, pero presenta diferencias por ciudad y dependencia escolar. La iniciativa social no se relaciona con el apoyo social parental en Antofagasta,

1 Trabajo realizado con un subsidio del Programa Bicentenario de Ciencia y Tecnología (PBCT), Chile y del Fondo de Apoyo a la Investigación (FAI) de la Universidad de los Andes.

2 Psicólogo. Facultad de Psicología Universidad del Desarrollo. Escuela de Psicología Universidad Pedro de Valdivia. Santiago, Chile. E-mail: martin_casassus@yahoo.com

3 Enfermera Universitaria. Máster en Salud Pública Universidad Carolina del Norte. Decana Facultad de Enfermería, Universidad San Sebastián, Santiago, Chile. E-mail: Macarena.valdes@uss.cl

4 Médico Psiquiatra. Máster en Salud Pública m/Salud Mental, Universidad Carolina del Norte. Director de Investigación, Universidad del Desarrollo. Jefe del Servicio de Psiquiatría Hospital del Salvador, Profesor Psiquiatría Universidad de los Andes. Santiago, Chile. E-mail: rflorenzano@udd.cl 5 Arquitecto. Máster en Matrimonio y Familia, Universidad de Navarra. Instituto de Ciencias de la Familia, Universidad de los Andes y Dirección de Desarrollo Académico, Universidad San Sebastián, Chile. E-mail: caceres.eugenio@gmail.com

6 Psicóloga. Facultad de Psicología, Universidad del Desarrollo, Becaria CONICYT Santiago, Chile. E-mail: maspillagah@udd.cl

7 Médico Cirujano, Magíster en Psicología de la Adolescencia, m/Biomédica m/Psicoeducación. Doctor () en Ciencias de la Educación. Jefe de Departamento de Ciclo Vital. División de prevención y control de Enfermedades, Ministerio de Salud, Chile. E-mail: silvia.santander@gmail.com 
pero sí en Santiago y Concepción. Indicadores de depresión son más altos en colegios particulares privados; los subvencionados presentan los menores puntajes en comportamiento antisocial.

Palabras clave: parentalidad, conductas de riesgo adolescente, estilos de crianza.

\begin{abstract}
Parenting styles influence adolescent's mental health. Previous work has corroborated the explicative model proposed by Brian Barber that correlates parental variables (psychological control, behavioral control, social support) with adolescent functional behaviors (depression, antisocial behavior, social initiative). In this study the model is applied in a representative sample of students in three Chilean cities with different administrative school systems. Results: the model fits in the overall sample, but presents differences by city and school system. Social initiative doesn't correlate with parental support in Antofagasta, but it does in Santiago and Concepción. Depressions indexes are higher in private schools; state subsidized schools present the lower scores in the antisocial behavior scale.
\end{abstract}

Key words: parenthood, adolescent risk behavior, parenting styles.

\title{
Introducción
}

Esta investigación corresponde a un trabajo de profundización de otros estudios realizados por el mismo equipo, en relación con la aplicación de un modelo de parentalidad y comportamiento adolescente planteado por Brian Barber (2002).

La interacción entre padres e hijos es una relación que afecta a ambos agentes sociales dentro del sistema familiar y puede constituir beneficios o daños potenciales para ambas partes. Por esto, la percepción de los estilos parentales de crianza se ha transformado en un importante tema de estudio. Con este objeto se han creado y aplicado instrumentos 
para evaluar formas de crianza y su repercusión en el desarrollo adolescente. Baumrind (1968) contrastó las tendencias de crianza permisivas con las autoritarias, concluyendo que el buen uso de la autoridad era una herramienta conveniente durante la niñez, pero que no cumple el mismo efecto en la adolescencia. Baumrind influenció gran parte de la investigación ulterior que siguió trabajando en base a estas variables categóricas, tal como aparece documentado en Vallejo, Osorno, Mazadiego (2008). Brian Barber (2002), en la Universidad de Kentucky, planteó un modelo en base a variables de Percepción de Estilos de Parentalidad (PEP) y otras en el área de la salud emocional y/o conductual adolescente, que operacionalizó en el cuestionario CNAP (Cross National Adolescent Program), validado transculturalmente por él y su equipo.

El modelo explicativo de la conducta adolescente planteado por Barber está constituido por tres variables PEP y tres de funcionamiento adolescente (FA), tal como aparece reflejado en Florenzano, Cáceres et al. (2009) y en Florenzano, Valdés, et al. (2009) donde se describen las variables utilizadas para crear este modelo. Las variables PEP son: el Control Conductual Parental (CCP), el Control Psicológico Coercitivo Parental (CPP) y el Apoyo Social Parental (ASP), las cuales interactúan con las variables de salud mental adolescente. El CCP se refiere al control disciplinario y al monitoreo de las actividades del adolescente por parte de los padres, por lo tanto suele ser un control explícito, que incluye, por ejemplo, con quién pasan su tiempo libre o qué hacen con él. Esta variable de percepción de parentalidad, se configura de esta forma, como un indicador no solo del control en sí mismo, sino que de la comunicación y confianza que existe en la relación paterno-filial, desde la perspectiva del adolescente.

El CPP corresponde al modo de control mediante una coerción psicológica ejemplificado en actitudes como retirar el cariño frente al desacuerdo con las figuras de cuidado o culpabilizar al adolescente, como una forma de hacer que estos se comporten conforme a las reglas de sus cuidadores (Florenzano, Cáceres, et al., 2009; Florenzano, Valdés, et al. 2009). Este tipo de control parental dificulta el desarrollo de la autonomía 
psicológica (Steinberg, 1990, en Betancourt \& Andrade, 2007), a la vez que se le ha asociado al desarrollo de comportamientos problemáticos (Palacios \& Andrade, 2008).

Adicionalmente, el ASP describe un reforzamiento de la individualidad del adolescente, así como un apoyo frente a su desenvolvimiento social (Florenzano, Cáceres, et al., 2009; Florenzano, Valdés, et al., 2009).

Los estudios de Barber y de este equipo han corroborado que la existencia de una adecuada ASP y bajo CPP, se asocia con puntajes más bajos en la escala de sintomatología depresiva, tanto en los adolescentes de 10 a 15 años, como de 15 a 19 años (Florenzano, Valdés, et al., 2009). Sin embargo, queda pendiente conocer cómo se ajusta el modelo a las distintas realidades e historias de interacciones que se tienen (viven) en diferentes realidades socioculturales, puesto que tal como muestra el VII Estudio Nacional de Drogas en Población Escolar del Consejo Nacional de Control de Estupefacientes ([CONACE], 2006), en Chile existen diferencias por ciudad en el consumo de drogas lícitas e ilícitas y en conductas de tipo antisocial. Dado lo anterior, podría esperarse que la diversidad sociocultural que existe entre las distintas zonas geográficas de dicho país y las diferentes realidades de socialización que se hallan en las distintas dependencias escolares, podrían ser un factor que influencie el comportamiento del modelo. De este modo resulta relevante establecer si el modelo, que ya presenta un buen ajuste a nivel de la capital, mantiene su consistencia en otras ciudades de Chile y si es transversal al tipo de dependencia escolar bajo la cual estudian los adolescentes.

Los trabajos de Barber establecen una relación con dirección negativa entre el ASP y la sintomatología depresiva (SD), así como entre el CCP y el comportamiento antisocial adolescente (CAA), mientras que se observa una relación positiva entre el ASP y la iniciativa social adolescente (ISA). Además, también existe una relación positiva entre el CPP, con la SD y CAA (Florenzano, Valdés, et al., 2009). Si bien los resultados de esas investigaciones son consistentes no contemplan una segmentación de los datos en sus análisis para analizar el fenómeno en la realidad socioeducativa 
diferente que viven los jóvenes. Esto resulta relevante, ya que tal como afirman Casullo y Liporace (2008) las condiciones educativas y económicas influyen en las variables de PEP. Para dar respuesta a cómo sería la influencia de dichas condiciones se realizó el estudio en dos ciudades representativas de distintas zonas geográficas de Chile (Antofagasta y Concepción), a la vez que se plantea un análisis a partir del tipo de dependencia del centro de educación que reciben los adolescentes.

La educación chilena en sus niveles preescolar, básico (ocho años) y medio (cuatro años) es administrada por un sistema mixto, con un rol conductor estatal, que incluye una operación descentralizada de la educación estatal o pública y un área de gestión privada. En tanto el Estado mantiene funciones normativas, evaluativas, de supervisión, de apoyo técnico, de financiamiento y control, las competencias de administración directa están descentralizadas, en la educación básica y media, en las municipalidades (entidades descentralizadas de la organización estatal) $\mathrm{y}$ en entidades privadas que pueden desarrollar sus actividades con formas libres de educación o con reconocimiento oficial del Estado, lo que significa ajustarse a requisitos legales y curriculares determinados. Según las estadísticas del Ministerio de Educación (2009), la matrícula total es de casi 3.600.000 alumnos que se distribuían en alrededor de 1.550.000 en la Dependencia Municipal (DM) (43\%), cerca de 1.720 .000 en la Dependencia Particular con Subvención Estatal (DPSE) (48\%) y 253.000 en la Dependencia Particular Pagada (DPP) (7\%). El 2\% restante corresponde a la "gestión delegada a corporaciones", no incluida en este reporte.

Considerando los antecedentes, este trabajo reporta cuáles son las diferencias entre realidades geográficas y socioeducativas en la Percepción de Estilos de Parentalidad y cómo inciden estas diferencias en la sintomatología adolescente, reflejada tanto en la conducta antisocial, como en una escala de sintomatología depresiva, a la vez que refieren su influencia en la iniciativa social adolescente, que se establece como un indicador de desarrollo positivo en esta etapa del desarrollo. 


\section{Método}

Se aplicó el instrumento CNAP-Plus, una adaptación chilena del CNAP descrito por Barber (2002). Dicha adaptación al castellano se realizó en reuniones de trabajo en conjunto con el autor, en Bellagio, Italia, en el 2006, y en las ciudades de Santiago y Concepción durante el 2007. El cuestionario consiste en un autorreporte y cuenta, entre otras, con escalas de Control Conductual, Control Psicológico, Apoyo Social Parental, Iniciativa Social, Depresión (Achenbach) y Comportamiento Antisocial (Achenbach), que son las variables que son sujetas a análisis en este estudio.

Se realizó un muestreo probabilístico en base al listado de colegios del Ministerio de Educación de Chile, estratificando por el nivel socioeconómico de la comuna en donde se encontraba el colegio y la dependencia a la cual pertenece cada establecimiento educacional. La recolección de datos se llevó a cabo en dos etapas. La primera en Santiago, en el 2007, y la segunda, en las otras dos ciudades, el año 2008. La aplicación de los cuestionarios se realizó en las aulas de los establecimientos educacionales seleccionados y fue realizado por sujetos entrenados en la aplicación del cuestionario CNAP-Plus.

La muestra está compuesta por 2.347 adolescentes escolarizados pertenecientes a las ciudades de Antofagasta (Región de Antofagasta), Santiago (Región Metropolitana) y Concepción (Región del Biobío). La elección de estas ciudades, si bien siguió un criterio de conveniencia, se realizó con el fin de lograr incluir realidades distintas a la de la capital del país, con otras características climáticas y con un predominio de diferente actividad económica.

Además, los colegios firmaron un consentimiento informado institucional en el cual se comprometió devolución de la información y los alumnos un asentimiento informado. El detalle del número de adolescentes encuestados por región, dependencia del establecimiento y sexo se presenta en la Tabla 1. 
Tabla 1

Distribución de la muestra por Género, dependencia del establecimiento educacional y Región de Chile

\begin{tabular}{llcc}
\hline & & Recuento & $\begin{array}{c}\text { \% del N de la } \\
\text { columna }\end{array}$ \\
\hline Género & Masculino & 1026 & $44,2 \%$ \\
& Femenino & 1296 & $55,8 \%$ \\
Tipo de & Municipales & 794 & $33,8 \%$ \\
& Particular & & \\
& subvencionado & 947 & $40,3 \%$ \\
& Particular pagado & 606 & $25,8 \%$ \\
Región de Chile & Antofagasta & 457 & $19,5 \%$ \\
& Metropolitana & 1445 & $61,6 \%$ \\
& Biobío & 445 & $19,0 \%$
\end{tabular}

Para el análisis de datos, se utilizaron tablas de frecuencias para la presentación de las características de la muestra y medidas de tendencia central, dispersión y forma. Luego se realizaron correlaciones de Pearson para la comprobación del modelo de Barber, estableciendo correlaciones entre las tres variables de salud mental adolescente (SD, CAA e ISA) y las tres variables de percepción de parentalidad (CCP, CPP y ASP), tanto en el análisis de la muestra total, como en la segmentación de la muestra. Finalmente, se realizó una comparación entre dependencias escolares y entre ciudades mediante Anova de una vía, con la corrección post hoc HDS de Tukey. Para todos los análisis estadísticos se utilizó una significancia máxima de 0,05 .

Es importante mencionar que el estudio fue aprobado por las comisiones de ética del Fondo de Apoyo a la Investigación (FAI) de la Universidad de los Andes y por el Programa Bicentenario de Ciencia y Tecnología (PBCT). 


\section{Resultados}

Un total de 2.347 adolescentes contestaron correctamente sus datos de identificación referente a la dependencia de su Colegio (DM, DPSE y DPP) y ciudad de Chile a la que pertenecían (Antofagasta, Santiago y Concepción). En la caracterización por sexo (hombre o mujer) se registraron 25 casos perdidos (ver Tabla 1).

La Tabla 2 muestra las medidas de tendencia central, dispersión y forma de las escalas analizadas. De los datos se desprende que las escalas que miden sintomatología (depresión y CAA) presentan una asimetría y una curtosis muy positiva, donde la mayoría de los casos se agrupa en bajas puntuaciones (ver Tabla 2).

Tabla 2

Estadísticos descriptivos de las escalas de Control Conductual, Control Psicológico, Apoyo social parental, Iniciativa Social, depresión y comportamiento antisocial

\begin{tabular}{|c|c|c|c|c|c|c|c|}
\hline & $\mathrm{N}$ & Mínimo & Máximo & Media & $\begin{array}{l}\text { Desv. } \\
\text { típ. }\end{array}$ & Asimetría & Curtosis \\
\hline $\begin{array}{l}\text { Control } \\
\text { Psicológico } \\
\text { Parental }\end{array}$ & 1966 & 0 & 32 & 8,88 & 5,904 &, 574 &,- 073 \\
\hline $\begin{array}{l}\text { Control } \\
\text { Conductual } \\
\text { Parental }\end{array}$ & 2087 & 10 & 30 & 22,39 & 4,586 &,- 223 &,- 597 \\
\hline $\begin{array}{l}\text { Apoyo } \\
\text { Social } \\
\text { Parental }\end{array}$ & 1934 & 0 & 40 & 28,32 & 9,020 &,- 761 &,- 096 \\
\hline $\begin{array}{l}\text { Iniciativa } \\
\text { Social }\end{array}$ & 2157 & 13 & 65 & 35,91 & 9,729 &, 153 &,- 222 \\
\hline $\begin{array}{l}\text { Depresión } \\
\text { Achenbach }\end{array}$ & 2287 & 0 & 10 & 2,19 & 2,250 & 1,218 & 1,212 \\
\hline $\begin{array}{l}\text { Compor- } \\
\text { tamiento } \\
\text { Antisocial }\end{array}$ & 2260 & 0 & 16 & 3,32 & 2,468 & 1,050 & 1,570 \\
\hline $\begin{array}{l}\text { N válido } \\
\text { (según lista) }\end{array}$ & 1599 & & & & & & \\
\hline
\end{tabular}


El análisis de la muestra total (Tabla 3) evidencia una relación estadísticamente significativa con dirección negativa entre el Apoyo Social Parental y la sintomatología depresiva. Así, a medida que existe un menor Apoyo Social Parental, existen más indicadores de sintomatología depresiva en los jóvenes. La percepción de un Control Conductual Parental consistente se correlaciona negativamente con los puntajes en la escala de depresión. Por su parte el Control Psicológico Parental se correlaciona de modo directamente proporcional con indicadores de sintomatología depresiva (ver Tabla 3), por lo que los modos de control culpabilizadores, coercitivos y fundados en la amenaza con el retiro del carińo, refieren un mayor desarrollo de sintomatología depresiva.

\section{Tabla 3}

Correlaciones de las escalas de Control Conductual, Control Psicológico, Apoyo social parental, Iniciativa Social, depresión y comportamiento antisocial en la muestra total

$\begin{array}{ccc}\text { Control } & \text { Control } & \text { Aceptación } \\ \text { Conductual } & \text { Psicológico } & \text { Biparental } \\ \text { Biparental } & \text { Biparental } & \end{array}$

\begin{tabular}{|c|c|c|c|c|}
\hline \multirow[t]{3}{*}{ Depresión } & $\begin{array}{l}\text { Correlación de } \\
\text { Pearson }\end{array}$ &,$- 227(* * *)$ &, $\left.298{ }^{(* * *}\right)$ &,$- 337(* * *)$ \\
\hline & Sig. (bilateral) & ,000 &, 000 & ,000 \\
\hline & $\mathrm{N}$ & 2043 & 1922 & 1893 \\
\hline \multirow[t]{3}{*}{$\begin{array}{l}\text { Comportamiento } \\
\text { Antisocial }\end{array}$} & $\begin{array}{l}\text { Correlación de } \\
\text { Pearson }\end{array}$ &,$- 266\left({ }^{* * *}\right)$ &, $242(* * *)$ &,$- 204(* * *)$ \\
\hline & Sig. (bilateral) & , 000 &, 000 &, 000 \\
\hline & $\mathrm{N}$ & 2017 & 1907 & 1878 \\
\hline \multirow[t]{3}{*}{ Iniciativa Social } & $\begin{array}{l}\text { Correlación de } \\
\text { Pearson }\end{array}$ &, $175\left(^{(* *}\right)$ &,- 025 &, $183\left(^{(* * *}\right)$ \\
\hline & Sig. (bilateral) & ,000 & ,283 &, 000 \\
\hline & $\mathrm{N}$ & 1928 & 1827 & 1805 \\
\hline
\end{tabular}

*** La correlación es altamente significativa $\mathrm{p}<0,001$

** La correlación es significativa al nivel 0,01 (bilateral).

* La correlación es significante al nivel 0,05 (bilateral). 
En el caso de la escala de Comportamiento Antisocial Adolescente se repite la misma tendencia que con la sintomatología depresiva, encontrándose correlaciones negativas significativas con el Control Conductual Parental y Apoyo Social Parental. Por lo tanto los adolescentes que presentan un Control Conductual Parental y Apoyo Social Parental consistentes, presentan puntajes más bajos en la escala de Comportamiento Antisocial Adolescente.

Por su parte el Control Psicológico Parental se correlaciona significativamente y con una dirección positiva, con la escala de Comportamiento Antisocial, por lo que la percepción de un alto CPP favorece la presencia de conductas de tipo antisocial (Tabla 3).

La escala de Iniciativa Social Adolescente se correlaciona de forma estadísticamente significativa y con dirección positiva con las escalas de Control Conductual Parental y Apoyo Social Parental. No se encuentra correlación entre esta escala y el Control Psicológico Parental (Tabla 3).

En el análisis por ciudad, destaca el hecho de que en Antofagasta la Iniciativa Social no se correlaciona con ninguna de las tres variables de parentalidad, por lo que ninguna de las variables de percepción de parentalidad promueve el desarrollo de la Iniciativa Social. Por su parte, en Santiago se encuentran correlaciones significativas con el Apoyo Social Parental y con el Control Conductual Parental.

En Santiago, las correlaciones estadísticamente significativas más fuertes se registran entre las escalas de Apoyo Social Parental y el Control Psicológico Parental con la escala de Depresión. En Concepción es donde se presentan los montos de correlación más bajos, siendo la correlación entre el Apoyo Social Parental y la Escala de Depresión la más fuerte (ver Tabla 4). 
Tabla 4

Correlaciones de Percepción de Estilos Parentales con variables de funcionamiento adolescente, por región

Ciudad de

Chile
Depresión Comportamiento Iniciativa

Achenbach Antisocial Social

Control

Antofagasta Psicológico Pearson ,228(***) ,296(***) ,074

Parental

\begin{tabular}{cccc}
$\begin{array}{c}\text { Sig. } \\
\text { (bilateral) }\end{array}$ &, 000 &, 000 &, 139 \\
$\mathrm{~N}$ & 409 & 408 & 402 \\
\hline
\end{tabular}

Control

Conductual Pearson $\quad-, 213\left({ }^{* * *}\right) \quad-, 259(* * *) \quad, 099$

Parental

$\begin{array}{lll}\begin{array}{c}\text { Sig. } \\ \text { (bilateral) }\end{array} & , 000 & , 000\end{array}$

\begin{tabular}{ccccc} 
& $\mathrm{N}$ & 404 & 403 & 394 \\
\hline $\begin{array}{c}\text { Apoyo Social } \\
\text { Parental }\end{array}$ & Pearson &,$- 213\left({ }^{* * *}\right)$ &,$- 203\left({ }^{* * *}\right)$ &,- 022
\end{tabular}

$\begin{array}{cccc}\begin{array}{c}\text { Sig. } \\ \text { (bilateral) }\end{array} & , 000 & , 000 & , 659\end{array}$

$\begin{array}{llll}\mathrm{N} & 405 & 404 & 398\end{array}$

\begin{tabular}{|c|c|c|c|c|c|}
\hline \multirow[t]{9}{*}{ Santiago } & $\begin{array}{c}\text { Control } \\
\text { Psicológico } \\
\text { Parental }\end{array}$ & Pearson &, $\left.351{ }^{(* * *}\right)$ &, $277(* * *)$ &,- 047 \\
\hline & & $\begin{array}{c}\text { Sig. } \\
\text { (bilateral) }\end{array}$ & ,000 &, 000 & ,128 \\
\hline & & $\mathrm{N}$ & 1134 & 1119 & 1063 \\
\hline & $\begin{array}{c}\text { Control } \\
\text { Conductual } \\
\text { Parental }\end{array}$ & Pearson &,$- 257\left({ }^{* * *}\right)$ &,$- 301\left(^{* * *}\right)$ &, $231(* * *)$ \\
\hline & & $\begin{array}{c}\text { Sig. } \\
\text { (bilateral) }\end{array}$ & ,000 &, 000 &, 000 \\
\hline & & $\mathrm{N}$ & 1246 & 1220 & 1158 \\
\hline & $\begin{array}{c}\text { Apoyo Social } \\
\text { Parental }\end{array}$ & Pearson &,$- 407(* * *)$ &,$- 254\left(^{* * *}\right)$ &, $271\left(^{* * *}\right)$ \\
\hline & & $\begin{array}{c}\text { Sig. } \\
\text { (bilateral) }\end{array}$ &, 000 &, 000 &, 000 \\
\hline & & $\mathrm{N}$ & 1119 & 1104 & 1053 \\
\hline
\end{tabular}




\section{Control}

\begin{tabular}{|c|c|c|c|c|c|}
\hline Concepción & $\begin{array}{c}\text { Psicológico } \\
\text { Parental }\end{array}$ & Pearson &, $213\left({ }^{* * *}\right)$ &, $142(* *)$ &,- 048 \\
\hline & & $\begin{array}{c}\text { Sig. } \\
\text { (bilateral) }\end{array}$ & ,000 & ,006 &, 365 \\
\hline & & $\mathrm{N}$ & 378 & 379 & 361 \\
\hline & $\begin{array}{c}\text { Control } \\
\text { Conductual } \\
\text { Parental }\end{array}$ & Pearson &,$- 149\left(^{* *}\right)$ &,$- 187(* * *)$ & ,078 \\
\hline & & $\begin{array}{c}\text { Sig. } \\
\text { (bilateral) }\end{array}$ & ,003 & ,000 & , 130 \\
\hline & & $\mathrm{N}$ & 392 & 393 & 375 \\
\hline & $\begin{array}{c}\text { Apoyo Social } \\
\text { Parental }\end{array}$ & Pearson &,$- 230(* * *)$ &,- 089 &, $145\left(^{* *}\right)$ \\
\hline & & $\begin{array}{c}\text { Sig. } \\
\text { (bilateral) }\end{array}$ & ,000 & ,088 & ,006 \\
\hline & & $\mathrm{N}$ & 368 & 369 & 353 \\
\hline
\end{tabular}

*** La correlación es altamente significativa $\mathrm{p}<0,001$

** La correlación es significativa al nivel 0,01 (bilateral).

* La correlación es significante al nivel 0,05 (bilateral).

El análisis según dependencia escolar (ver Tabla 5), muestra correlaciones más fuertes asociadas a la Escala de Depresión. En la Dependencia Municipal la correlación más fuerte se presenta entre Depresión y el Apoyo Social Parental, donde a mayor percepción de ASP, hay menor presencia de indicadores de síntomas depresivos. Por otra parte, una alta percepción de Control Psicológico se asocia a una mayor presencia de síntomas depresivos. La Escala de Comportamiento Antisocial Adolescente presenta una correlación negativa con el Apoyo Social Parental y esta misma variable (ASP) es la que mejor se correlaciona con la escala de Iniciativa Social Adolescente (Tabla 5). 
Tabla 5

Correlaciones de Percepción de Estilos Parentales con variables de funcionamiento adolescente, por tipo de establecimiento educacional

\begin{tabular}{|c|c|c|c|c|c|}
\hline $\begin{array}{c}\text { Tipo de } \\
\text { Establecimiento } \\
\text { Educacional }\end{array}$ & & & $\begin{array}{l}\text { Depresión } \\
\text { Achenbach }\end{array}$ & $\begin{array}{c}\text { Comportamiento } \\
\text { Antisocial }\end{array}$ & $\begin{array}{c}\text { Iniciativa } \\
\text { Social }\end{array}$ \\
\hline \multirow[t]{9}{*}{ Municipales } & $\begin{array}{l}\text { Control } \\
\text { Psicológico } \\
\text { Parental }\end{array}$ & Pearson &, $335\left(^{* * *}\right)$ &, $256\left(^{* * *}\right)$ & ,016 \\
\hline & & $\begin{array}{c}\text { Sig. } \\
\text { (bilateral) }\end{array}$ &, 000 & ,000 & ,696 \\
\hline & & $\mathrm{N}$ & 610 & 604 & 581 \\
\hline & $\begin{array}{c}\text { Control } \\
\text { Conductual } \\
\text { Parental }\end{array}$ & Pearson &,$- 217(* * *)$ &,$- 271(* * *)$ &, $\left.1811^{* * *}\right)$ \\
\hline & & $\begin{array}{c}\text { Sig. } \\
\text { (bilateral) }\end{array}$ &, 000 &, 000 &, 000 \\
\hline & & $\mathrm{N}$ & 665 & 654 & 624 \\
\hline & $\begin{array}{c}\text { Apoyo } \\
\text { Social } \\
\text { Parental }\end{array}$ & Pearson &,$- 380\left(^{* * *}\right)$ &,$- 285(* * *)$ &, $221\left(^{* * *}\right)$ \\
\hline & & $\begin{array}{l}\text { Sig. } \\
\text { (bilateral) }\end{array}$ & ,000 & ,000 & ,000 \\
\hline & & $\mathrm{N}$ & 600 & 597 & 573 \\
\hline \multirow[t]{9}{*}{ Subvencionados } & $\begin{array}{c}\text { Control } \\
\text { Psicológico } \\
\text { Parental }\end{array}$ & Pearson &, $\left.230{ }^{(* * *}\right)$ &, $236\left(^{* * *}\right)$ &,- 012 \\
\hline & & $\begin{array}{l}\text { Sig. } \\
\text { (bilateral) }\end{array}$ &, 000 & ,000 &, 745 \\
\hline & & $\mathrm{N}$ & 795 & 796 & 758 \\
\hline & $\begin{array}{c}\text { Control } \\
\text { Conductual } \\
\text { Parental }\end{array}$ & Pearson &,$- 183\left({ }^{* * *}\right)$ &,$- 270\left(^{* * *}\right)$ &, $\left.140{ }^{(* * *}\right)$ \\
\hline & & $\begin{array}{c}\text { Sig. } \\
\text { (bilateral) }\end{array}$ &, 000 & ,000 &, 000 \\
\hline & & $\mathrm{N}$ & 825 & 824 & 787 \\
\hline & $\begin{array}{c}\text { Apoyo } \\
\text { Social } \\
\text { Parental }\end{array}$ & Pearson &,$- 326\left(^{* * *}\right)$ &,$- 206\left(^{* * *}\right)$ &, $106\left(^{* *}\right)$ \\
\hline & & $\begin{array}{c}\text { Sig. } \\
\text { (bilateral) }\end{array}$ & ,000 &, 000 & ,004 \\
\hline & & $\mathrm{N}$ & 779 & 778 & 746 \\
\hline
\end{tabular}




\begin{tabular}{|c|c|c|c|c|c|}
\hline \multirow[t]{9}{*}{$\begin{array}{l}\text { Particular } \\
\text { Pagados }\end{array}$} & $\begin{array}{c}\text { Control } \\
\text { Psicológico } \\
\text { Parental }\end{array}$ & Pearson &, $344(* * *)$ &, $270\left(^{* * *}\right)$ &,- 058 \\
\hline & & $\begin{array}{c}\text { Sig. } \\
\text { (bilateral) }\end{array}$ & ,000 & ,000 & , 198 \\
\hline & & $\mathrm{N}$ & 516 & 506 & 487 \\
\hline & $\begin{array}{c}\text { Control } \\
\text { Conductual } \\
\text { Parental }\end{array}$ & Pearson &,$- 294(* * *)$ &,$- 271(* * *)$ &, $179\left(^{* * *}\right)$ \\
\hline & & $\begin{array}{c}\text { Sig. } \\
\text { (bilateral) }\end{array}$ & ,000 & ,000 & ,000 \\
\hline & & $\mathrm{N}$ & 552 & 538 & 516 \\
\hline & $\begin{array}{c}\text { Apoyo Social } \\
\text { Parental }\end{array}$ & Pearson &,$- 268\left(^{* * *}\right)$ &,$- 111\left(^{*}\right)$ &, $199\left(^{* * *}\right)$ \\
\hline & & $\begin{array}{c}\text { Sig. } \\
\text { (bilateral) }\end{array}$ & ,000 & ,013 &, 000 \\
\hline & & $\mathrm{N}$ & 513 & 502 & 485 \\
\hline
\end{tabular}

*** La correlación es altamente significativa $\mathrm{p}<0,001$

** La correlación es significativa al nivel 0,01 (bilateral).

* La correlación es significante al nivel 0,05 (bilateral).

En la Dependencia Particular con Subvención Estatal la correlación más fuerte se encuentra entre la Escala de Depresión y la de Apoyo Social Parental. En la escala de Comportamiento Antisocial, la asociación más fuerte se presenta con la percepción de un Control Conductual Parental y esta última es, además, la que mejor se correlaciona con la escala de Iniciativa Social Adolescente (Tabla 5).

En la Dependencia Particular Pagada se encuentra que la Escala de Depresión se correlaciona significativamente con la percepción del Control Psicológico. En el caso del Comportamiento Antisocial Adolescente se encuentran correlaciones con una fuerza casi equivalente con las escalas de Control Psicológico y Control Conductual, aunque con direcciones diferentes: en CPP la dirección es positiva y en el CCP es negativa (Tabla 5).

Respecto a las diferencias en el comportamiento de las escalas por ciudad, se puede observar que la percepción de CCP presenta estabilidad en todas las regiones, mientras que todas las otras variables, tanto de funcionamiento personal como de PEP, presentan diferencias al menos entre 
dos de las ciudades (ver Tabla 6). El análisis Post Hoc de Tukey muestra que en el Control Psicológico Parental hay diferencias entre las ciudades de Antofagasta y Santiago ( $\mathrm{p}=0,007)$ con una mayor puntuación en Antofagasta; en el ASP no se observan diferencias en la corrección de Tukey; en la escala de ISA se encuentran diferencias entre Antofagasta con las ciudades de Santiago y de Concepción ( $\mathrm{p}=0,000$ y $\mathrm{p}=0,021$ respectivamente, siendo más altos los puntajes en la ciudad de Santiago y más bajos en Concepción). En la escala de depresión solo existen diferencias entre las ciudades de Santiago y Concepción, con puntajes más altos en la primera de estas $(\mathrm{p}=0,020)$ y en la escala de CAA las diferencias se presentan entre Antofagasta y Santiago ( $\mathrm{p}$ $=0,000)$, también siendo esta última la de puntuación más alta.

\section{Tabla 6}

\section{Diferencias en las escalas por Ciudad y por Dependencia Escolar}

\begin{tabular}{|c|c|c|c|c|c|c|c|c|c|c|c|}
\hline & & \multicolumn{5}{|c|}{ Entre Ciudades } & \multicolumn{5}{|c|}{ Entre Dependencias } \\
\hline & & $\begin{array}{l}\text { Suma de } \\
\text { cuadrados }\end{array}$ & $\mathrm{gl}$ & $\begin{array}{c}\text { Media } \\
\text { cuadrática }\end{array}$ & $\mathrm{F}$ & Sig. & $\begin{array}{l}\text { Suma de } \\
\text { cuadrados }\end{array}$ & gl & $\begin{array}{c}\text { Media } \\
\text { cuadrática }\end{array}$ & $\mathrm{F}$ & Sig. \\
\hline \multirow{3}{*}{$\begin{array}{c}\text { Control } \\
\text { Psicológico } \\
\text { Parental }\end{array}$} & Inter-grupos & 325,154 & 2 & 162,577 & 4,681 & 0,009 & 401,746 & 2 & 200,873 & 5,791 & 0,003 \\
\hline & Intra-grupos & 68171,468 & 1963 & 34,728 & & & 68094,876 & 1963 & 34,689 & & \\
\hline & Total & 68496,623 & 1965 & & & & 68496,623 & 1965 & & & \\
\hline \multirow{3}{*}{$\begin{array}{c}\text { Control } \\
\text { Conductual } \\
\text { Parental }\end{array}$} & Inter-grupos & 85,138 & 2 & 42,569 & 2,026 & 0,132 & 355,939 & 2 & 177,969 & 8,525 & 0 \\
\hline & Intra-grupos & 43777,127 & 2084 & 21,006 & & & 43506,327 & 2084 & 20,876 & & \\
\hline & Total & 43862,265 & 2086 & & & & 43862,265 & 2086 & & & \\
\hline \multirow{3}{*}{$\begin{array}{l}\text { Apoyo Social } \\
\text { Parental }\end{array}$} & Inter-grupos & 510,329 & 2 & 255,164 & 3,143 & 0,043 & 2108,978 & 2 & 1054,489 & 13,124 & 0 \\
\hline & Intra-grupos & 156755,984 & 1931 & 81,179 & & & 155157,335 & 1931 & 80,351 & & \\
\hline & Total & 157266,313 & 1933 & & & & 157266,313 & 1933 & & & \\
\hline \multirow[t]{3}{*}{ Iniciativa Social } & Inter-grupos & 2934,417 & 2 & 1467,209 & 15,713 & 0 & 5623,67 & 2 & 2811,835 & 30,522 & 0 \\
\hline & Intra-grupos & 201125,197 & 2154 & 93,373 & & & 198435,944 & 2154 & 92,124 & & \\
\hline & Total & 204059,614 & 2156 & & & & 204059,614 & 2156 & & & \\
\hline \multirow{3}{*}{$\begin{array}{l}\text { Depresión } \\
\text { Achenbach }\end{array}$} & Inter-grupos & 43,281 & 2 & 21,641 & 4,285 & 0,014 & 51,971 & 2 & 25,986 & 5,15 & 0,006 \\
\hline & Intra-grupos & 11533,87 & 2284 & 5,05 & & & 11525,18 & 2284 & 5,046 & & \\
\hline & Total & 11577,152 & 2286 & & & & 11577,152 & 2286 & & & \\
\hline \multirow{3}{*}{$\begin{array}{c}\text { Comportamiento } \\
\text { Antisocial }\end{array}$} & Inter-grupos & 119,562 & 2 & 59,781 & 9,89 & 0 & 109,46 & 2 & 54,73 & 9,048 & 0 \\
\hline & Intra-grupos & 13642,864 & 2257 & 6,045 & & & 13652,966 & 2257 & 6,049 & & \\
\hline & Total & 13762,427 & 2259 & & & & 13762,427 & 2259 & & & \\
\hline
\end{tabular}

*** Los datos son altamente significativa $\mathrm{p}<0,001$

** Los datos son significativa al nivel 0,01 (bilateral).

* Los datos son significante al nivel 0,05 (bilateral). 
En cuanto a la dependencia escolar se observan diferencias en todas las variables de Percepción de Estilos Parentales y de salud mental adolescente (Tabla 6). En el Control Psicológico Parental, estas muestran puntuaciones más altas en la Dependencia Municipal con respecto a la Dependencia Particular Pagada ( $\mathrm{p}=0,020)$ y más altas de la Dependencia Subvencionada sobre la Particular Pagada $(\mathrm{p}=0,003)$.

En la escala de Control Conductual Parental se observan puntajes más bajos en la Dependencia Particular Pagada y en la Dependencia Subvencionada, respecto a la Dependencia Municipal $(\mathrm{p}=0,0001$ y $\mathrm{p}=$ 0,021 respectivamente).

En la escala de Apoyo Social Parental, la Dependencia Particular Pagada tiene puntajes más altos que la Subvencionada $(\mathrm{p}=0,002)$ y la Municipal $(\mathrm{p}=0,000)$.

Dentro de las escalas de funcionamiento adolescente, la escala de Iniciativa Social Adolescente, muestra puntajes más altos en la Dependencia Particular Pagada sobre la Subvencionada y la Municipal ( $p=0,0001$ en ambos casos). La escala de Depresión solo presenta diferencias entre la Dependencia Municipal y la Dependencia Particular Pagada ( $p=0,004)$, siendo más bajos los puntajes de estos últimos. En la escala de Comportamiento Antisocial Adolescente, los establecimientos con Dependencia Particular con Subvención Estatal, presentan puntajes más bajos que las otras dos dependencias ( $\mathrm{p}=0,005$ con la DM y $\mathrm{p}=0,000$ con la DPP).

\section{Discusión}

Del análisis de los resultados en las tres ciudades incluidas en la investigación (Antofagasta, Santiago y Concepción) se desprende que, tal como plantea el modelo estudiado, cuando los adolescentes perciben de sus figuras parentales un alto apoyo para exploración social y una alta percepción de respeto a su individualidad, desarrollan menos atributos propios de la sintomatología depresiva, menos conductas de tipo antisocial y una mejor Iniciativa Social. Estos hallazgos son congruentes con lo planteado por Barber (2002) y por las investigaciones previas del equipo de este estudio. 
La confirmación de que la percepción adolescente de cuidadores hostigadores, que ejercen su autoridad a través de mecanismos culpógenos y que amenazan con el retiro del cariño frente a una mala acción se constituye como un factor de riesgo en el desarrollo de estos síntomas, es un hallazgo relevante, dada la transversalidad de esa tendencia entre ciudades y contextos escolares. Un resultado que coincide con Barber (2002) y Delgado, Jiménez, Sánchez-Queija y López (2007) es que el Control Psicológico Parental coercitivo es un factor claramente nocivo en la salud mental del adolescente. Esto plantea un desafío en la generación de programas de conductas parentales saludables, los cuales podrían implementarse dentro de las políticas en salud, a través del trabajo en consultorios y centros de salud familiar, o dentro de las políticas educativas, al incluir talleres para padres dentro de los establecimientos educacionales, respecto a modos de ejercicio de la autoridad.

A pesar de que los resultados tienen una dirección muy clara, cuando se realizan los análisis conjuntos de las tres ciudades, al dividirlas, se encuentran algunas diferencias relevantes. En Antofagasta, la Iniciativa Social Adolescente no se ve influenciada por ninguna de las tres variables de Percepción de Estilos Parentales, por lo que el desarrollo de esta variable de salud mental adolescente debe estar influido por otros factores, que deberán explorarse en futuros análisis. Por su parte, en Concepción las correlaciones se mantienen, pero con menor fuerza que en Santiago. Estos hallazgos sugieren que la ciudad en la que viven los adolescentes es una variable que interviene en el modelo de estilos parentales, cuyas razones deberán ser explicadas en futuros estudios. Una hipótesis que podría ser motivo de trabajo en futuras aproximaciones es que en urbes menos pobladas, las interacciones sociales fuera del ámbito familiar adquieren una mayor influencia que en las grandes ciudades, a la hora de inducir conductas protectoras y de riesgo en los adolescentes, teniendo siempre presente que cualquier modelo explicativo debe observarse como un fenómeno multicausal. Complementario a lo anterior, es importante destacar dentro del análisis por ciudad que la mayor presencia de atributos de Sintomatología Depresiva y de Comportamiento Antisocial se registró en la ciudad de Santiago. Sin embargo, estas diferencias solo 
resultan significativas entre las ciudades de Santiago y Concepción para la escala de depresión, y entre las ciudades de Santiago y Antofagasta en el Comportamiento Antisocial.

La dependencia del establecimiento escolar también es un factor que afecta el modelo de parentalidad y comportamiento adolescente planteado por Barber. El análisis profundo de estos resultados se complejiza al revisar las estadísticas respecto a cómo se componen socioeconómicamente, las distintas dependencias escolares. Según datos de un estudio realizado por Espínola, Claro y Walker (2009), encargado por el Ministerio de Educación, respecto a un Diagnóstico de la Educación Media, un 75\% de los alumnos matriculados en colegios de dependencia particular pagada, corresponde al quinto quintil de ingresos, mientras que un 64,2\% de los matriculados en escuelas de dependencia municipal corresponde a los dos primeros quintiles. Por lo tanto, las diferentes dependencias no solo dan cuenta de una realidad educativa diferente, sino de toda una complejidad socioeducativa, que incluye tanto el mundo interaccional directo de los adolescentes con sus padres como un conjunto de variables que van desde las expectativas individuales y colectivas, a su repertorio e historia de interacciones y aprendizajes previos.

Las asociaciones más fuertes siempre aparecieron ligadas a la escala de depresión, tanto en la Dependencia Municipal, como en la Dependencia Particular con Subvención Estatal y Dependencia Particular Pagada. En las Dependencias Municipal y Subvencionada, un bajo Apoyo Social Parental es el factor que más se correlaciona con el desarrollo de sintomatología depresiva, mientras que en la Dependencia Particular Pagada, se establece la asociación más fuerte con la percepción de un alto Control Psicológico Parental. Es importante resaltar que los puntajes más bajos en la escala de Control Psicológico se encuentran en la Dependencia Particular Pagada, por lo que el ejercicio de este tipo de autoridad y control parece ser un factor que afecta más a los adolescentes pertenecientes a este tipo de establecimientos y realidad socioeducativa.

Se afirma entonces que la realidad contextual en la que viven los adolescentes incide significativamente en la aplicabilidad del modelo explicativo de Barber, al menos respecto a la ciudad en la que viven y a la 
realidad socioeducativa que comparten. Si bien el peso de estas variables macro-contextuales analizadas no alcanza a desarticular el modelo en su análisis global (sin distinguir ciudades ni dependencias escolares), se puede observar que los contrastes entre las distintas realidades de nuestro país representan una condición a tomar en cuenta a la hora de elaborar programas de intervención a nivel nacional.

No obstante los resultados de la investigación son consistentes, existen limitantes importantes de mencionar para la revisión de los alcances del estudio. La primera de ellas corresponde a que la estrategia de muestreo utilizada no fue proporcional a la dependencia de los colegios, por lo que la distribución regional de los colegios no se halla bien representada en la muestra. Por otra parte, se reconoce como una limitación la ausencia de seguimiento de la muestra. Por lo tanto, para futuros estudios sería importante realizar varias mediciones en el tiempo, idealmente intercaladas por programas dirigidos a cambiar estilos parentales "patologizantes", con el fin de determinar si una intervención terapéutica o una psicoeducación a padres, luego de los 15 ańos del adolescente, logra mejorar los indicadores de salud mental.

Dentro de los análisis que no han sido realizados en este reporte, que resultarían importantes, se destacan las diferencias entre las familias biparentales y mono-parentales, así como las diferencias del comportamiento del modelo diferenciando entre la percepción materna y paterna, como también el incorporar la perspectiva de género en la segmentación de los datos para el análisis, como la realizada por Mestre, Tur, Samper, Nácher y Cortés (2007) quienes encontraron importantes diferencias. Además, queda pendiente la construcción de modelos multivariados y de relaciones no lineales, que podrían entregar más información respecto al fenómeno en estudio.

Finalmente, es importante mencionar que la amplia difusión que puede darse a los resultados descritos puede establecerse como una referencia válida para la definición de políticas públicas relativas al factor protector que representa la familia en torno a las conductas de riesgo adolescente y/o el desarrollo de sintomatología. De esta forma (y tal como se ha expuesto anteriormente), se pueden usar los espacios de socialización de 
los adolescentes (como son sus escuelas) para ofrecer programas destinados a los cuidadores, en relación con las distintas maneras de ejercicio de la autoridad. Respecto a lo anterior, es importante aclarar que, en ningún caso, los resultados de este estudio pretenden configurar una "receta" para un buen ejercicio parental, pero sí se considera que la socialización de la información contenida en este documento puede ser de gran ayuda para elaborar programas de promoción de salud familiar, iniciativas que pueden gestionarse desde el sector público y privado, al incorporar estas variables dentro de los programas de promoción de salud (lo que puede darse en espacios tanto educativos como laborales), a partir de la evidencia del repertorio de interacciones del propio contexto sociocultural y de toda su particular diversidad.

\section{Referencias}

Barber, B. (2002). Intrusive parenting: How psychological control affects children and adolescents. Washington, DC: American Psychological Association Press.

Baumrind, D. (1968). Authoritarian vs. authoritative parental control. Adolescence, 3, 255-272.

Betancourt, D. \& Andrade, P. (2007) Escala de percepción parental en niños. Investigación Multidisciplinaria Universitaria, Año 6, 6, 26-34.

Casullo, M. \& Liporace, M. (2008) Percepción de estilos e inconsistencias parentales en adolescentes argentinos. Estud. psicol. (Campinas). 25(1), 3-9.

Consejo Nacional de Control de Estupefacientes [CONACE] (2006). Séptimo Estudio Nacional de Drogas en población escolar. Recuperado el 11 de febrero de 2010 desde http://www.conacedrogas.cl/inicio/pdf/ informe_ejecutivo_Estudio_Escolares_Conace2007_16-06-2008. pdf

Delgado, A., Jiménez, A., Sánchez-Queija, I. \& López, F. (2007). Estilos Educativos Materno y Paterno: Evaluación y relación con el ajuste adolescente. Murcia, España. Anales de Psicología, Servicio de Publicaciones de la Universidad de Murcia, 23(1), 49-56. ISSN edición web (www.um.es/analesps): 1695-2294. 
Espínola, V., Claro, J. P. \& Walker, H. (2009) Lineamientos estratégicos para la discusión de una política de mediano plazo para la educación media: Diagnóstico de la educación media. Apartado I (1 de 3). Facultad de Educación, Universidad Diego Portales; Ministerio de Educación, Gobierno de Chile, 2009. Recuperado el 10 de diciembre de 2011 desde http://w3app.mineduc.cl/mineduc/ded/documentos/Lineamientos_Educacion_Media___Diagnostico.pdf.

Florenzano, R., Cáceres, E., Valdés, M., Calderón, S., Santander, S. \& Casassus, M. (2009). Conductas de Riesgo, síntomas depresivos, auto y heteroagresión en una muestra de adolescentes escolarizados en la Región Metropolitana de Santiago. Revista Chilena de NeuroPsiquiatría, 47(1), 24-33.

Florenzano, R., Valdés, M., Cáceres, E., Casassus, M., Sandoval, A. \& Santander, S. (2009) The Perception of Parental Relationship: A Comparison of Adolescents Older and Younger Than 15 Years of Age. Revista chilena de pediatría, 80(6): 520-527. Recuperado el 16 de febrero de 2010 desde: http://www.scielo.cl/scielo.php?script=sci_ arttext\&pid=S0370-41062009000600004\&lng=es. doi: $10.4067 /$ S0370-41062009000600004

Mestre, M., Tur, A., Samper, P., Nácher, M. \& Cortés, M. (2007). Estilos de crianza en la adolescencia y su relación con el comportamiento prosocial. Revista Latinoamericana de Psicología, 39(2), 211-225.

Ministerio de Educación, Gobierno de Chile (2009). Distribución de la Matrícula por Dependencia y Área Geográfica año 2008. Recuperado el 11 de mayo de 2010 desde http://w3app.mineduc.cl/DedPublico/educacion_en_cifras

Palacios, J. \& Andrade, P. (2008). Influencia de las prácticas parentales en las conductas problema en adolescentes. Investigación Multidisciplinaria Universitaria. Año 7, 7, 7-18.

Vallejo, A., Osorno, R. \& Mazadiego, T. (2008) Estilos parentales y sintomatología depresiva en una muestra de adolescentes veracruzanos. Enseñanza e Investigación en Psicología, 13(1), 91-105. Universidad Veracruzana, Xalapa, México. Recuperado el 12 de abril de 2010 desde http://redalyc.uaemex.mx/src/inicio/ArtPdfRed. jsp?iCve $=29213108$

Fecha de recepción: 27 de julio de 2011.

Fecha de aceptación: 13 de diciembre de 2011. 
